1. 中国科学院自动化研究所脑网络组研究中心, 北京 100190 ;

2. 中国科学院自动化研究所, 模式识别国家重点实验室, 北京 100190

*联系人, Email: jiangtz@nlpr.ia.ac.cn

收稿日期：2019-10-23; 接受日期：2019-12-26; 网络版发表日期：2020-03-16

国家重点研发计划(批准号：2017YFB1002502)、国家自然科学基金国际合作重点项目(批准号：31620103905)、国家自然科学基金面上项目 (批准号: 61971420)和中国科学院战略性先导科技专项(批准号: XDB32030200)资助

摘要随着脑影像技术和计算技术等领域的发展, 基于活体影像的脑科学研究呈爆发式增长, 并受到来自多种不 同学科领域研究人员的广泛关注, 其中包括神经科学、临床医学、心理学以及工程技术等学科领域. 近年来, 不 同学科交叉融合形成了新的研究方向和领域, 这也是脑科学发展独具学科特色的研究模式. 对于活体人脑结构和 功能信号的检测技术而言, 许多技术在基础科研和临床上已经得到比较广泛的应用, 包括多模态磁共振技术、脑 电及脑磁技术等. 与此同时, 脑科学研究在时间和空间分辨率上对脑信号检测技术提出了更高的要求, 由此产生 的数据为后期的数据挖据、建模分析等技术带来了更多的挑战. 为了更好地描述从多时空尺度研究大脑的模式, 本团队在国际上提出了 “脑网络组学”的概念. 本文系统回顾了目前基于影像的脑科学研究在非侵入性宏观影像 层面有代表性的技术，同时强调了多模态多种技术的联合应用，即“脑网络组学”的研究背景和内涵. 文章最后对 脑网络组学研究发展作出展望.

关键词脑网络组学, 脑网络组图谱, 脑网络, 神经影像, 转化医学

人脑被普遍认为是复杂并且高效的信息处理系 统, 也是迄今为止人类对自身认识最少的器官. 人脑的 复杂性不仅表现在其复杂的解剖结构, 更表现在其灵 活多样的认知功能. 早期的神经科学研究主要集中在 离体的细胞和神经元培养和操作等微观层面, 以及纯 粹的行为学和心理学等宏观层面. 最近 20 年来, 活体 脑影像技术的发展，尤其是功能影像技术的突飞猛进， 使得在体无损地检测脑功能活动成为可能. 该技术不 仅可以在人脑处于静息状态(resting state)下研究其不 受外界干扰的自发活动，而且在被试应对外界刺激的 情形(task state)下也能同步记录其功能活动, 这些技术 的应用有效地连接起上述微观和宏观两个层面的研 究. 另外, 随着计算技术的发展, 科研人员可以更灵活 地基于多种模态影像的脑结构和功能活动数据进行分 析和建模, 因此, 成像技术和计算技术的结合, 为脑结 构和功能的研究提供了强有力的工具.

尽管众多的影像技术和计算方法不断涌现出来, 但由于脑本身的复杂性, 只有部分技术方法对脑科学 的研究产生了深远影响. 并且, 随着人脑这个“黑匣 子”逐步打开, 人们也意识到对自身脑的认知才刚刚开

引用格式: 左年明, 蒋田仔. 多模态人脑影像与脑网络组学研究. 中国科学: 生命科学, 2020, 50: 1296-1308 Zuo N M, Jiang T Z. Multi-modal human brain mapping and Brainnetome study (in Chinese). Sci Sin Vitae, 2020, 50: 1296-1308, doi: 10.1360/SSV2019-0228 
始. 本文对近年来热门的脑科学研究技术进行了总结 回顾，这些技术随着不断的升级将有可能在接下来的 脑科学研究中发挥更重要的作用，这些技术包括不同 时间-空间尺度的活体脑成像技术，例如多模态磁共振 技术、脑电/脑磁技术，而且还包括最新的脑影像分析 和建模方法, 例如脑网络组图谱构建、脑网络分析方 法及转化医学应用研究等. 由于该领域受到广泛关注, 所以涉及的方法众多，其他的活体脑成像技术还包括 功能近红外成像 ${ }^{[1]}$ 、光电同步脑活动成像技术 ${ }^{[2]}$ 等, 所测量的信号在神经机理上与上述技术基本相似，所 以不逐一赘述.

\section{1 多模态跨尺度脑影像技术}

\section{1 多模态磁共振成像}

磁共振成像(magnetic resonance imaging, MRI)技 术作为一种无创的高时间-空间分辨率的成像技术，已 经在临床上得到广泛应用。临床MRI成像主要依靠人 体组织内水分子的氢核在外加磁场下产生核磁共振的 现象, 从而获取组织内水分子的分布, 进而间接地反映 不同组织间的对比度. 在实际的MRI应用中, 根据不同 组织的对比度特性而设计出的特殊的射频脉冲序列, 能反映出人体组织结构、功能代谢(血氧水平变化)及 神经纤维结构等信息.

根据不同的成像脉冲序列(pulse sequence), 研究 人员还设计出针对特定脑组织及信号的成像模式. 脑 功能活动的刻画是 20 世纪末磁共振技术对脑科学的重 要贡献. 脑内神经元活动会引起相应脑区的血氧水平 变化, 因此通过测量血氧水平依赖(blood oxygenationlevel dependent, BOLD)信号, 即可以间接地反应脑的 神经活动 ${ }^{[3]}$. 这种基于 $B O L D$ 的功能磁共振测量(functional MRI, fMRI) 在静息状态 ${ }^{[4]}$ 和任务状态 ${ }^{[5]}$ 的脑活 动记录都已经得到大量应用. 另外, 动脉自旋标记(arterial spin labeling, ASL)作为一种反映组织血流灌注 情况的fMRI技术也逐渐受到关注. ASL利用血液中水 分子作为内源性示踪剂分别生成标记图像和对照图 像, 通过减影技术得到血流灌注变化敏感的图像, 目前 比较成熟的为Dai等人 ${ }^{[6]}$ 基于伪连续式动脉自旋标记 (pseudo continuous arterial spin labeling, pCASL)技术. 随着近年来磁共振成像序列技术的完善，ASL技术已 经逐渐成为临床刻画脑血流灌注异常的重要手段 ${ }^{[7,8]}$.
值得注意的是, BOLD信号和血流灌注水平都是反映 神经元活动过程血氧供应和消耗的动态变化, 因此只 是间接地反应脑神经功能活动, 该响应变化通常用血 液动力学响应函数(hemodynamic response function, HRF)表示, 最新的研究也表明, 该函数形式在不同年 龄段以及不同的脑区都有差异 ${ }^{[9]}$. 由于涉及时间序列 的数据采集, 头动因素是必须考虑和弥补的因素 ${ }^{[10]}$, 也有研究表明, 头动大小也与个体的功能活动相关 ${ }^{[1]}$.

对于脑结构的成像, 除了常规的 $\mathrm{T} 1$ 和 $\mathrm{T} 2$ 加权成像, 基于水分子的受阻扩散现象描述白质纤维结构也受到 广泛关注，这种使用扩散加权脉冲序列的方法称为扩 散加权成像 (diffusion-weighted imaging, DWI) ${ }^{[12]}$. 该 方法早期用于敏感地检测脑卒中的病灶，后续的研究 人员结合不同方向的扩散加权信号, 利用不同的建模 方法, 可以在宏观上模拟得到纤维的排布，这些方法 包括基于简单的高斯扩散模型(即扩散张量成像, diffusion tensor imaging, DTI), 以及基于反卷积或球调 和基函数展开的高角度分辨率成像建模(high angular resolution diffusion imaging, HARDI $)^{[13]}$, 此外, 基于 HARDI的采样模式还包括反映神经排布方向和密度 的多元结构建模方式 ${ }^{[14]}$. 相对于DTI方法, 后两类方法 可以在纤维交叉的白质脑区得到更真实的纤维方向重 构 $^{[13]}$. 常规的DTI重建理论上只需要 6 个梯度方向和一 个参考图像(b0), 但是HARDI的图像采集模式通常需 要更多的梯度方向甚至更多类别的扩散磁场强度(也 称为 $b$-value), 所以通常会延长采集时间.

磁共振脑成像技术近年来取得了许多进展, 如多 频带平面回波成像(multiband echo planer imaging, MB-EPI $)^{[15]}$ 技术可以实现多层同时扫描，大幅减少了 扫描时间. 另外, 在时空分辨率上的提高, 使得灰质皮 层分层的fMRI 扫描(layer fMRI) ${ }^{[16,17]}$ 也成为可能. MRI 技术与其他成像技术联合形成的多模态成像技术也是 一个重要的研究方向, 比如PET-MRI技术(其中PET为 positron emission tomography), 能够整合PET提供的人 体生理代谢、分子信息和MRI提供的解剖形态及功能 信息, 实现PET及MRI成像设备的优势互补, 对神经、 心血管、肿瘤等疾病的研究及临床诊断具有重要意 $义^{[18]}$. 另外还有 fMRI-EEG设备, 可以同步记录血氧代 谢水平活动以及神经元电活动 ${ }^{[19,20]}$. 我国在磁共振成 像硬件技术开发方面一直较为欠缺, 而在图像处理及 神经科学领域的应用一直与国际同行保持相同水平. 


\section{2 脑电图及脑磁图技术}

脑电图(electroencephalogrphy, EEG)和脑磁图 (magnetoencephalography, MEG)均是无创性快速探测 大脑神经元电生理活动的神经功能检测技术(检测速 度在毫秒级或者亚毫秒级), 可由放置于头皮表面的传 感器记录由大脑神经元突触后电位电流活动产生的微 弱电势和磁场信号变化.

1929年, 德国人Hans Berger第一次在人的头皮上 记录到了 $\mathrm{EEG}^{[21]}$. 从此EEG逐渐成为人们探索大脑活 动的重要工具. 目前, EEG检测时间分辨率可达毫秒 级别 (数千赫兹), 主要包括诱发电位及事件相关电 位、自发脑电、睡眠脑电图等多种形式. 其硬件和软 件上也拥有了可以和MRI, MEG等模态兼容的仪器和 完整的分析算法, 以及应用于群体认知、神经营销、 脑机接口、医疗康复等多领域的便携式、无线化产 品. MEG最早始于1969年由Cohen博士 ${ }^{[22]}$ 在美国麻省 理工大学进行的脑磁场图像测量. 随着计算机的飞速 发展和各种应用软件的开发, MEG装置发生了由单信 道到多信道、从局部观测到全头部观测的质的变化. 目前, 传感器阵列的信道总数已达到306个, 具有可靠 的磁场屏蔽系统及灵敏的磁场探测系统，可同时高速 采集整个大脑的瞬态神经活动数据. 由于在电场传导 过程中大脑和头皮之间的头骨和其软组织并不会扭曲 及衰减磁信号, 因此, MEG相对于EEG能对脑内信号 源做到更高度精确的空间定位 (精度在数毫米之内). 结合MEG快速的时间分辨率(数千赫兹)和精确的空间 定位能力, MEG可以用于测定脑功能信号精细的传递 过程. 因此, MEG对于临床诊断、脑外科手术前的手 术计划制定以及脑的基础研究等都具有十分重要的 意义.

在外界刺激输入下，脑部产生的时间锁定的电生 理活动, 即诱发/事件相关的电位(event-related potential, ERP)及磁场(event-related field, ERF)活动, 是人 们在EEG和MEG研究中主要关注的信号之一. 这种活 动往往通过多次重复实验, 利用平均叠加技术从头颖 表面记录大脑诱发电位/磁场来反映刺激或认知过程 中大脑的神经电生理改变. 因为 $\mathrm{ERP} / \mathrm{ERF}$ 与认知过程 有密切关系, 故被认为是研究心理活动的重要工具, 取得了很多重要的研究成果. 除了ERP/ERF之外, EEG，MEG信号还有很多有节律的振荡 ${ }^{[23]}$. 尽管在很
长一段时间内这些振荡波动被人们认为是噪声或者是 脑活动的“副产物”[24]，往往通过叠加平均被极力消除， 但是近十多年来, 越来越多的研究开始重视这部分节 律信号. 通过大量动物研究已发现, 这些节律波动与 神经元的放电活动以及神经元群的同步振荡有 关 ${ }^{[25,26]}$, 通过能量-频率谱分析、时频分析、功能连接 及有效连接等分析方法对振荡信号的研究可以促进更 深入地理解认知过程涌现的神经生理基础, 并且获得 多种精神及神经疾病(如癫㾁、注意力不足活动症、 精神分裂症等)的生物标记 ${ }^{[27,28]}$.

目前，EEG和MEG已凭借良好的时间分辨率和日 益成熟的信号分析技术, 成为刻画脑功能连接、探索 认知功能涌现的脑功能活动机制、理解大量精神及神 经疾病的病理生理学基础的重要技术. 尽管已有一些 有力证据证明, $\mathrm{EEG} / \mathrm{MEG}$ 的振荡和神经元的放电及局 部场电位的振荡相关, 但是EEG/MEG信号的具体神经 机制还不是特别明确，例如各种疾病状态中已发现的 $E R P / E R F$ 以及节律的异常究竟来源于怎样的神经活动 异常? 不同认知状态中振荡节律及同步性的变化究竟 反映神经网络怎样的信息变化? 这些问题直接影响对 研究结果科学意义的理解和解读, 并阻碍了研究结果 向其他模态研究的迁移和向临床应用的成果转化. 信 号神经机制的不明确也直接影响到了EEG/MEG信号 与其他模态(例如fMRI、功能近红外成像、正电子发 射断层成像等)的融合, 目前尽管有各类融合概念和方 法的提出, 但是还只是尝试阶段.

\section{2 脑网络组学及脑网络组图谱}

\section{1 脑网络组学}

脑网络组学(Brainnetomics) ${ }^{[29]}$ 是以脑网络为基本 单元的组学, 研究内容包括利用各种成像技术及电生 理技术在宏观及微观尺度上建立人脑和动物脑的脑 区、神经元群或神经元之间的连接图(脑网络), 在此 基础上研究脑网络拓扑结构、脑网络的动力学属性、 脑功能及功能异常的脑网络表征、脑网络的遗传基 础, 并对脑网络进行建模和仿真, 以及搭建实现这些目 标所需的超级计算平台. 在此之前, 美国印第安纳大学 Sporns等人 ${ }^{[30]}$ 提出了“人脑连接组”概念, 旨在强调绘 制全脑结构连接网络的重要性，尽管该概念随后被推 广到功能网络的研究 ${ }^{[31]}$, 但是相比于“脑网络组学”仍 
然存在诸多不足, 包括研究的方式(时间/空间的多重 特性) 以及动态特征的表述 (发育老化过程及疾病的演 变)的局限. 脑网络组学的研究目标是从脑网络的连接 模式及其演变规律阐明脑的工作机理及脑疾病的发生 和发展机制, 为研究人脑内部复杂的信息处理过程与 高效的组织模式提供有效的途径, 为理解脑的信息处 理过程及脑的高级功能开辟新途径.

脑网络组学首次强调了脑网络分析中对网络节点 (脑区)和边(连接)定义的重要性, 其中, 脑区作为脑功 能基本工作单元(在不同尺度)的定义, 更是后续脑网 络分析的基础, 倘若不同功能脑区之间边界划分模糊, 后续的脑网络分析必然缺乏神经科学基础.

\section{2 脑网络组图谱}

脑图谱一直以来都是研究脑结构和功能及脑疾病 不可或缺的基础. 从文艺复兴时期的著名解剖学家维 萨里对大脑结构进行详细的描述开始, 大量的神经解 剖描述、图片和影像陆续出现. 随着技术的不断进步 和研究的不断深入, 各式各样的脑图谱层出不穷, 人 类脑图谱已经由早期的印刷版二维脑图谱发展到现在 的数字化三维、四维脑图谱; 由基于标本断面切片数 据发展到基于活体影像学数据构建的图谱; 由仅具有 个体脑解剖结构信息的单一图谱到包含群体解剖结构 及功能信息的多模态脑图谱 ${ }^{[22 ~ 34]}$. 在不同的时期, 出 现了一些里程碑式的脑图谱, 包括布罗德曼(Brodmann)脑图谱 ${ }^{[35]}$ 、Von Economo and Koskinas脑图 谱 $^{[36]}$ 、Talairach立体脑图谱 ${ }^{[37]}$ 、于利希细胞构筑脑图 谱 ${ }^{[38]}$ 等. 脑图谱的出现和不断完善对脑科学基础及临 床研究具有重要的意义. 但是, 由于人脑脑区数量众 多、个体变异明显, 加之目前对其各区域亚区数目及 其边界和连接模式也尚未完全明确, 缺乏精细的脑图 谱, 很大程度上限制了对脑功能及功能障碍病理机制 的认识. 为改变这一现状, 解决目前脑科学和脑疾病 研究的迫切需求, 针对现有脑图谱存在的本质问题, 中国科学院自动化研究所脑网络组研究中心团队 ${ }^{[39]}$ 突破传统脑图谱绘制的瓶颈, 提出利用脑解剖和功能 连接信息对脑区进行精细划分的图谱绘制方法, 成功 绘制出全新的人类脑网络组图谱.

脑网络组图谱(第一版)包括246个精细脑区亚区, 而且脑区亚区间的多模态连接模式比传统的Brodmann图谱精细4 5倍, 具有客观精准的边界定位. 本团
队 ${ }^{[39,40]}$ 第一次建立了宏观尺度上的活体全脑连接图 谱, 并对每个亚区进行了细致的功能描述. 脑网络组图 谱的绘制突破传统解剖学构建脑图谱的方式, 提出了 利用脑连接信息(基于扩散磁共振成像构建的白质纤 维连接并进行纤维束聚类分析)建立脑网络组图谱的 思想, 建立了脑网络组图谱构建所需脑亚区划分的新 理论和新方法. 脑网络组图谱适合于脑疾病和脑网络 研究, 具有明确的生物学意义. 首版脑网络组图谱已 经在脑网络组图谱门户网站(http://atlas.brainnetome. org)上, 以在线和离线软件的方式提供给相关研究领 域的基础与临床研究人员使用 ${ }^{[41]}$ (图1). 脑网络组图谱 发布之后, 引起了国内外的广泛关注, 被国际著名神经 解剖学家、澳大利亚科学院院士Paxinos ${ }^{[42]}$ 撰文特别 评述, 认为脑网络组图谱揭开了脑图谱研究的新篇章, 欧盟脑计划将它作为代表性的人脑图谱纳入其神经信 息平台, 被国际上脑成像分析主流软件推荐使用, 并入 选中国科学院院士和中国工程院院士投票评选的 “2016年中国十大科技进展新闻”、2016年度“中国十 大医学进展”, 以及2018年“中国科学院改革开放四十 周年40项代表性成果”.

脑网络组图谱能够提供每个亚区的解剖和功能连 接模式, 从而明确每个亚区的组织模式及功能意义, 这 将加深对于人类精神和心理活动的认识, 为理解人脑 结构和功能开辟了新途径, 并对未来类脑智能系统的 设计提供了重要启示, 也将为神经及精神疾病的新一 代诊断、治疗技术奠定基础, 并为脑中风损伤区域及 癫㾁病灶的定位、神经外科手术中的脑胶质瘤精确切 除等提供帮助, 提高诊断质量与治疗效果; 此外, 脑网 络组图谱的研究也会引发新技术和新设备的研 发 ${ }^{[29,43 \sim 46]}$. 虽然脑网络组图谱已经获得了国际同行广 泛认可, 但还有很多需要继续解决的问题, 比如脑网 络组图谱个体差异测量的信效度 ${ }^{[47,48]}$, 这是图谱个体 化(包括个体化定位和诊疗等需求)在临床上应用的必 经之路. 另外, 目前的脑网络组图谱是基于正常成年人 的数据计算得到, 而人脑在不同的年龄阶段一从胎 儿在母体前后, 到贞幼儿、儿童青少年、青年、中年 及老年等, 都会发生结构和功能的变化, 这使得针对 不同年龄段的图谱的研究变得非常必要 ${ }^{[49,50]}$. 另外, 脑图谱的功能验证, 无论是在脑疾病上的验证, 还是 在模式动物上的验证, 到目前仍然是一个开放的问题.

脑灰质结构的分区目前是影像神经科学的研究热 


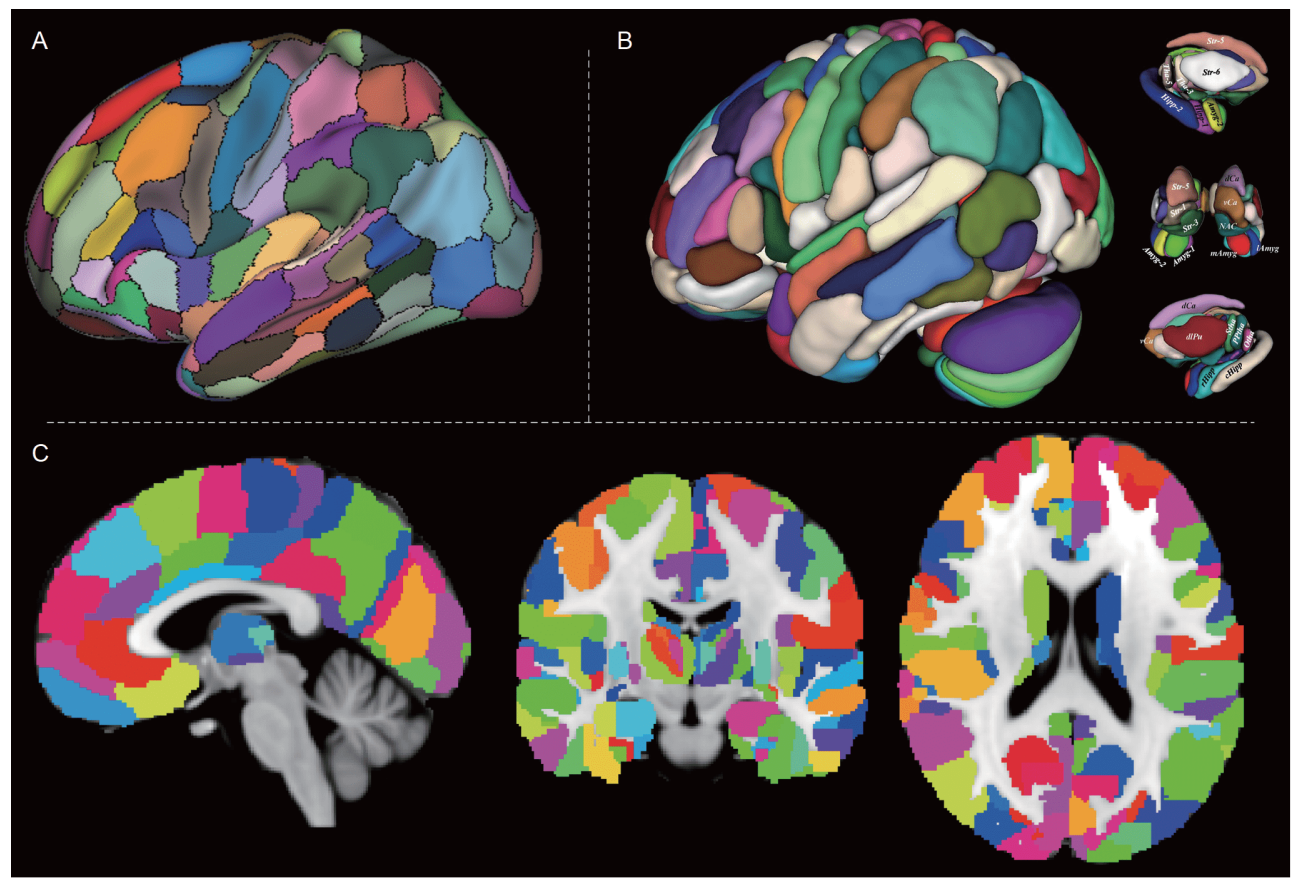

图 1 基于脑连接信息的脑网络组图谱 ${ }^{[39]}$. A: 灰质皮层表面绘制; B: 三维体绘制; C: 三维空间分别在矢状位、冠状位和轴状 位的三张截图

Figure 1 Connectivity-based Brainnetome atlas ${ }^{[39]}$ in different views. A: Surface rendering of the parcellations; B: 3D volume rendering; C: three slices in the sagittal view, coronal view and axial view, respectively

点之一. 最近几年出现的脑图谱中, 除了上述基于白质 纤维连接的图谱, 还有基于 $\mathrm{BOLD}$ 信号及功能连接聚

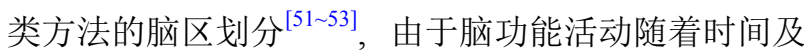
认知状态会发生动态改变，所以这种分区方式的稳定 性目前没有得到完全认可 ${ }^{[54]}$. 另外，也有直接基于脑

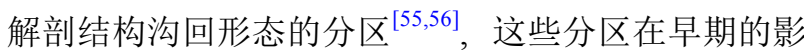
像学分析中得到了广泛应用, 但是缺少功能及功能连 接等信息. 最近也有研究人员提出了从灰质皮层的脑 区划分到头皮位置(如EEG/fNIRS等头皮位置测量方 法)的脑区映射方法 ${ }^{[57]}$.

\section{3 脑网络组学分析方法简介}

脑网络分析是以脑图谱定义的网络节点为基础结 合图论的脑结构和功能建模分析. 中国科学院自动化 研究所的蒋田仔团队 ${ }^{[58]}$ 于 2004 年率先引入图论提出 了不同节点共同作用的脑网络的概念，随后脑功能网 络和解剖结构网络的研究成为热点. 英国剑桥大学的 Bullmore团队 ${ }^{[59]} 2005$ 年构建了基于静息态fMRI的全 脑功能网络; 朱朝喆团队 ${ }^{[60]} 2009$ 年构建了基于近红外 脑功能成像(functional near-infrared spectroscopy,
fNIRS)的静息态脑功能网络. 基于EEG/MEG也可以在 探头间或者通过溯源算法在脑内各区域之间构建功能 网络 ${ }^{[61]}$. 贺永等人 ${ }^{[62]}$ 采用结构磁共振成像, 构建了基 于人脑皮层厚度相关的结构网络; 基于扩散磁共振成 像, Hagmann等人 ${ }^{[63]}$ 构建了个体水平的白质纤维结构 网络. 构建了网络连接之后, 基于图论的理论为脑网 络研究提供了系统性的新思路. 研究人员发现, 正常 成年人大脑网络具有 “小世界”属性( small-worldness)、模块化属性(modularity)、高级认知功能脑区 存在核心节点(hubness)等现象 ${ }^{[64]}$. 这种结构和功能的 拓扑属性使人脑能够高效地完成信息的分化和整合, 也为人们在系统水平上理解大脑是如何工作的提供了 独特的视角. 同时, 图论方法被广泛用于研究网络属性

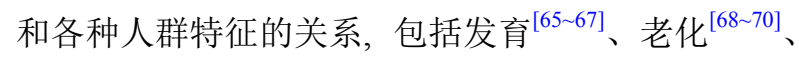
智商 ${ }^{[71,72]}$ 、工作记忆 ${ }^{[73,74]}$ 、性别效应 ${ }^{[75,76]}$ 、基因效 应 $^{[77 \sim 79]}$ 等.

我国研究者在神经影像计算软件的研发与推广 上, 也走在了国际最前沿, 开发了多个具有国际影响力 算法库和工具包, 为神经影像的人脑连接组研究提供 了重要的算法和软件平台, 得到了国际研究者的高度 
认可. 中国科学院自动化研究所脑网络组研究中心自 主开发了系列软件，包括基于磁共振影像的脑网络组 图谱构建软件 ${ }^{[41}$ (图2A)、脑图谱分区查看软件(图 $2 \mathrm{~B})$ 、功能磁共振分析软件BRANT ${ }^{[80]}$ 、扩散磁共振 分析软件DiffusionKit ${ }^{[13]}$, 另外还有北京师范大学开发 的神经影像脑网络可视化BrainNet Viewer 软件 ${ }^{[81]}$ 、静 息态磁共振数据分析软件 $\mathrm{REST}^{[82]}$ 和DPARSF ${ }^{[83]}$, 以及 中国科学院心理研究所开发的 $\mathrm{CCS}^{[84]}$ 和DPABI软件 ${ }^{[85]}$ 等. EEG脑信号分析方面，电子科技大学开发了参考电 极标准化技术REST工具包 ${ }^{[86]}$, 可以配合EEG专有工具 包EEGLAB使用 ${ }^{[87]}$ ，另外该团队还开发了EEG和fMRI 信号融合的脑网络分析工具包 ${ }^{[88]}$.

在脑网络分析领域，目前国内的相关团队已经处 于国际一流的水平，无论是算法开发设计、软件系统 平台，还是论文的引用率等，都获得了世界同行的认 可. 该领域的后续发展，将会集中在多模态多尺度的 数据融合脑网络分析方面，综合利用不同时间和空间 尺度的脑结构和功能信息, 结合最新的机器学习方法,
迅速准确地刻画脑的结构变化和功能动态活动.

\section{4 基于脑网络组学的转化医学}

上述多模态脑影像技术，结合脑网络组学理论的 发展, 对神经科学和临床医学都起到了很大的促进作 用. 在基础神经科学方面, 多模态磁共振成像技术已 经成功刻画出人脑结构和功能网络的许多固有网络特 性 ${ }^{[89]}$, 包括脑灰质皮层结构和功能网络的“小世界”属

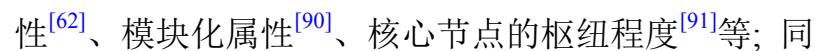
时，研究人员也发现了脑白质连接网络的年龄、性别 差异 ${ }^{[68]}$ 以及白质网络的效率与智商的关系 ${ }^{[71]}$. 针对正 常人的发育、发展、老化等全生命周期过程的脑结构 和功能变化(虽然大部分研究基于横向数据的研究). 研究人员也发现，脑区之间不同的相互作用形式也能 反映上述生命周期的不同过程，例如儿童发育过程中 网络模块化的形成 ${ }^{[65]}$, 这种区域差异性在老化过程中 减弱 ${ }^{[92]}$, 多需求网络(multiple demand network)的功能 连接模式能有效地反映老化的进程，该网络连接模式

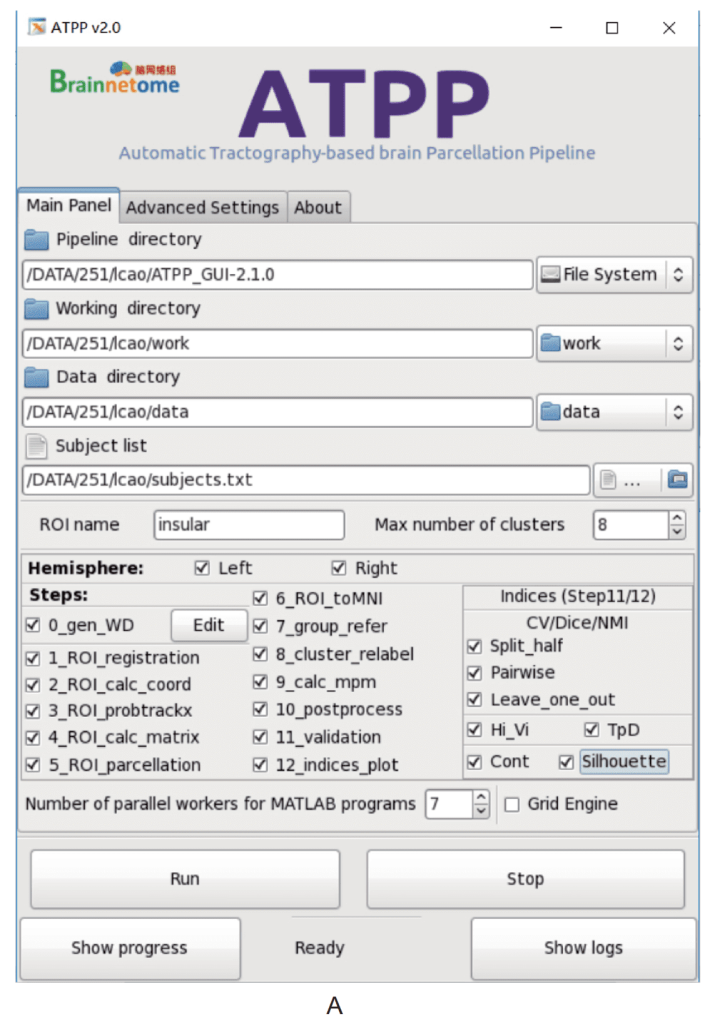

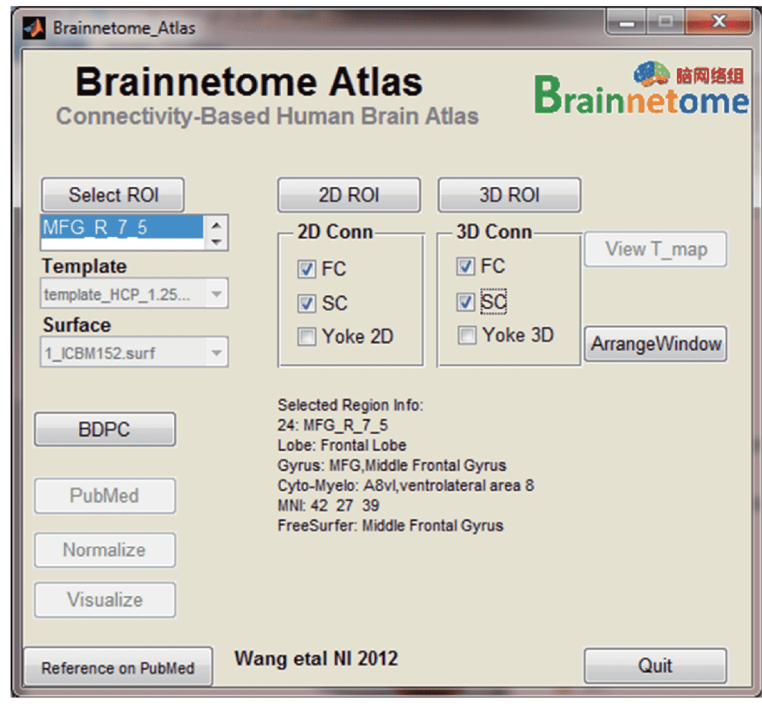

B

图 2 脑网络组图谱软件. A: 基于磁共振影像的脑网络组图谱构建软件 ${ }^{[41]}$; B: 脑图谱分区查看软件

Figure 2 The softwares for Brainnetome atlas. A: The software for constructing the Brainnetome atlas based on MRI data ${ }^{[41]}$; B: the Brainnetome atlas viewer 
退化慢的老年人其智商也退化得较慢 ${ }^{[93]}$. 因此, 人脑 老化过程在不同的个体上进程也有差异，该过程也与 脑网络的维持(maintenance) ${ }^{[94]}$ 及代偿 (compensation) ${ }^{[95]}$ 等特性有关. 进一步地, 研究人员也利用脑网络 的相关理论定量地刻画了人脑在同类型任务不同难度 情形下, 脑网络属性变化与任务能力的关系 ${ }^{[73,74]}$, 以及 脑网络在不同认知状态下转换的灵活性度量 ${ }^{[96]}$. 作为 在时间上可以实时采集脑活动信号的工具, EEG/MEG 可以被用于听觉、视觉、运动、语言、记忆、情绪、 睡眠、脑发育等众多研究领域. 例如，Michalareas等 人 ${ }^{[97]}$ 利用溯源计算后的MEG信号，通过Granger causality指标度量视觉皮层26个区域之间的功能连接, 并 利用功能连接的方向信息首次在人脑中刻画出视觉系 统的功能层级结构, 该层级结构包含很多人脑特有的 脑区，并发现在自下而上的前向通路信息传递主要以 gamma节律的活动为主，而自上而下的反馈通路信息 传递主要以alpha-beta节律活动为主.

除了上述基于正常人的基础神经科学研究，近年 来基于影像的脑网络的研究方法在临床神经精神疾病 中也取得了系列重要进展. 例如精神分裂症机理的失 连接假说 ${ }^{[98]}$ 、阿尔茨海默病(Alzheimer's disease)长程 连接减少现象 ${ }^{[99,100]}$ 、抑有症病人的额叶-边缘系统的 连接异常现象 ${ }^{[101]}$ 、视觉障碍(如盲人等)患者的视觉 皮层修剪缺失 ${ }^{[102]}$ 、功能连接代偿 ${ }^{[103]}$ 等. 另外, 在疾病 的诊断和分类判断应用中，脑网络技术借助最近的机 器学习方法也体现出诸多优势, 包括认知障碍疾病(阿 尔茨海默病及不同程度的认知障碍) 的分类诊断 ${ }^{[104]}$, 以及精神分裂症 ${ }^{[105]}$ 、孤独症 ${ }^{[106]}$ 、多动症等的分类判 断 ${ }^{[107]}$ 和昏迷病人促醒概率的预测判断 ${ }^{[108]}$ (图3), 以及 基于脑影像的年龄 ${ }^{[109]}$ 和性别 ${ }^{[110]}$ 的判断. 基于EEG/ MEG等的研究发现，诸如精神分裂症、自闭症等多种 神经精神疾病具有异常的节律振荡 ${ }^{[23]}$, 提示这些疾病 神经网络中兴奋性-抑制性活动出现失衡, 可能成为这 些疾病诊断的生物标记. 另外, EEG/MEG已广泛应用 于癫痫疾病的诊断、分类判别以及至病灶的定位中. 特别是基于MEG的高频振荡(high-frequency oscillations, HFO; > $90 \mathrm{~Hz}$ )溯源定位分析技术. 已有研究发 现，基于 $\mathrm{HFO}$ 定位区域执行病灶切除的患者术后康复 情况理想, 个别没有良好康复的患者被发现存在HFO 源定位区未被完全切除的现象 ${ }^{[111]}$. 因此, HFO目前被 越来越多的人认为是癫㾁至病灶的生物标记. EEG由
于其简单舒适、成本低的特点还被大量用于睡眠疾病 诊断和睡眠监测、麻醉深度监测、意识障碍、脑死亡 评定等领域. 例如，白杨等人 ${ }^{[112]}$ 利用EEG观测到意识 障碍患者在接受脊髓电刺激后脑活动出现振荡频率和 功能连接的改变, 并且其变化情况是刺激频率特异的, 这方面的工作可能会对脊髓电刺激治疗的频率选择产 生借鉴价值.

在基于多模态脑影像的转化医学研究中, 我国国 内相关主题的研究学者已经与世界水平持平, 在某些 领域甚至已经超过国际同行. 重要原因之一是我国的 人口基数大, 病例资源数据比较丰富; 同时, 政府早已 认识到各种脑疾病造成的沉重社会负担，因此各种相 关的脑影像和脑疾病的研究项目资助比较及时. 接下 来的研究需要从以下两个方面继续加强: (i) 侧重于 神经科学和临床医学的机制研究, 不能仅仅依赖数据 样本优势发现现象, 还需要研究病理背后的机制; (ii) 侧重于认知及发展的预测, 包括疾病的发展趋势判断 及预后判断、正常人发育及老化过程的预测及干预效 果评估, 争取在病症出现之前做到提前发现和干预.

\section{3 总结与展望}

基于多模态神经影像的脑网络组学已经取得了系 列重要进展, 但这些成果离真正实现临床应用还有一 段距离. 比如最常用的BOLD fMRI信号, 前文已经说 过它可间接反映神经元的功能活动, 但这两者之间的 具体关系仍然没有完全刻画清楚，目前发现它是随年 龄、认知状态及不同脑区等变化的 ${ }^{[9]}$, 这种现象称为 神经血管耦合 (neurovascular coupling) ${ }^{[113]}$. 为了实时 检测这两种信号, 脑网络组研究中心开发了同步光电 脑活动检测设备Near infrared spectroscopy \& Electroencephalography $(\mathrm{NEG})^{[114]}$, 通过该技术本团队 ${ }^{[2,115]}$ 初 步定量揭示了人脑视觉皮层处理不同视觉对比度刺激 时的神经血管耦合关系. 另外, 个体化定量计算是临床 精准医学应用的必经之路, 目前基于影像分析的定量 结论，包括图谱及基于图谱的脑网络分析，本质上是 基于群组的结论(包括差异检验或相关性分析等), 因 此个体化分析将是后续研究的重要主题之一. 同时, 目前的临床神经影像的数据种类比较多, 包括解剖结 构、血流标记、神经递质信息, 以及功能时间序列及 跟踪随访等, 多模态数据的融合分析可以综合利用这 


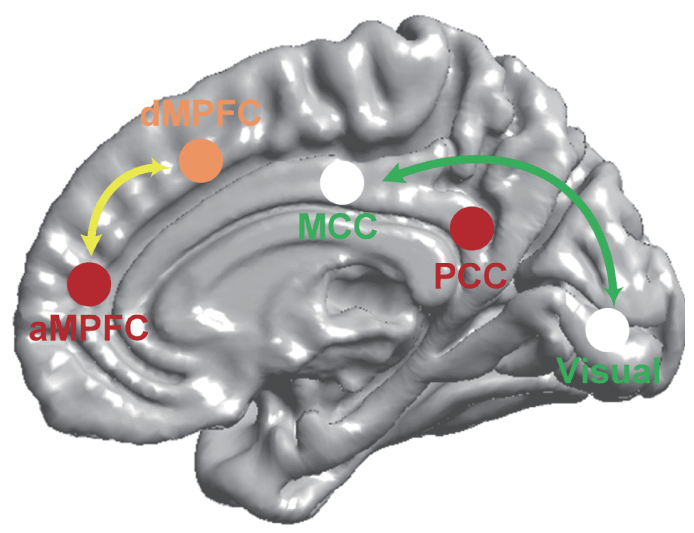

图 3 针对昏迷病人意识恢复的预测, 基于功能磁共振的功 能连接的预测准确率达到 $88 \%$, 对预测贡献最大的脑区包括 前部和背部内侧前额叶、扣带回中部皮层、后扣带皮层以 及视觉皮层, 以及图中所示功能连接 ${ }^{[188]}$

Figure 3 The accuracy of discriminating patients who would later recover consciousness and those who would not was around $88 \%$, based on the resting state fMRI. The brain regions with the highest importance in the prediction were located at anterior and dorsal medial prefrontal cortex, middle cingulate cortex, posterior cingulate cortex and visual cortex, as well as the connections shown in the figure ${ }^{[108]}$

些影像信息, 这也是目前的研究难点和热点之一, 除了 计算方法的研究, 数据的规范性和有效性也是该领域 重要的基础问题. 最后需要强调的是成像技术本身, 成像的时间-空间的精度是个永恒的话题，尤其是对于 临床应用, 快速诊断是临床追求的目标. 目前该方向有 几类发展趋势, 首先是快速成像, 例如磁共振成像中除
了硬件和序列的改进, 还包括基于机器学习的降采样 重建 ${ }^{[116,117]}$; 其次是小型化, 大型的医疗设备通常对使 用环境有较高的要求从而限制了使用范围，近年来移 动式的磁共振设备已经逐步开始推向临床使用 ${ }^{[118,119]}$, 脑网络组研究中心也研发推出了便携式的fNIRS成像 设备 ${ }^{[120]}$.

多模态影像技术及基于该技术的脑网络组学研 究, 无论在基础神经科学研究领域, 还是在临床医学的 应用研究中, 都已经成为主要的研究领域之一. 随着对 脑科学研究的深入细化, 脑科学的研究逐渐延伸出很 多分支, 从基因到细胞, 从神经元到功能柱, 从脑区到 脑网络再到行为学, 每个子领域都汇聚了不同交叉学 科背景的研究人员, 而随着成像技术和计算技术的迅 猛发展，上述这些领域又逐渐出现交叉，例如从神经 元的放电机理的模拟到行为决策的仿真 ${ }^{[121]}$. 后续的研 究将会持续贯穿在本文着重阐述的相关主题, 包括更 高时间空间分辨率脑结构和功能信号的获取技术(尤 其是在体的非侵入式方式), 以及多模态的信号的获取 和融合; 脑网络组图谱的进一步细化分区以及基于多 模态数据的个体化评估模型的构建; 脑网络组图谱的 生物学基础验证(包括基于疾病模型或基于模式动物 的验证); 不同模态脑网络的构建和融合, 以及脑网络 的动态分析; 基于脑图谱和脑网络的临床疾病研究、 个体化诊疗和预后判断; 基于脑图谱的脑功能解析及 面向仿脑计算的仿真模拟等.

致谢在论文写作过程中, 中国科学院自动化研究所森令仲博士、宋明博士、张玉瑾博士, 北京师范大学贺永博士等 人参与讨论并提供素材, 中国科学院自动化研究所研究助理马丽莹和姜晥清帮忙文字和图片校对修改, 在此一并致谢.

\section{参考文献}

1 Zhang Y, Tan F, Xu X, et al. Multiregional functional near-infrared spectroscopy reveals globally symmetrical and frequency-specific patterns of superficial interference. Biomed Opt Express, 2015, 6: 2786

2 Si J, Zhang X, Li Y, et al. Correlation between electrical and hemodynamic responses during visual stimulation with graded contrasts. J Biomed Opt, 2016, 21: 091315

3 Logothetis N K. What we can do and what we cannot do with fMRI. Nature, 2008, 453: 869-878

4 Buckner R L, Krienen F M, Yeo B T T. Opportunities and limitations of intrinsic functional connectivity MRI. Nat Neurosci, 2013, 16: 832-837

5 Gore J C. Principles and practice of functional MRI of the human brain. J Clin Invest, 2003, 112: 4-9

6 Dai W, Garcia D, de Bazelaire C, et al. Continuous flow-driven inversion for arterial spin labeling using pulsed radio frequency and gradient fields. Magn Reson Med, 2008, 60: 1488-1497

7 Telischak N A, Detre J A, Zaharchuk G. Arterial spin labeling MRI: Clinical applications in the brain. J Magn Reson Imag, 2015, 41: 1165-1180 
8 Haller S, Zaharchuk G, Thomas D L, et al. Arterial spin labeling perfusion of the brain: Emerging clinical applications. Radiology, 2016, 281: $337-356$

9 West K L, Zuppichini M D, Turner M P, et al. BOLD hemodynamic response function changes significantly with healthy aging. NeuroImage, 2019, 188: 198-207

10 Power J D, Barnes K A, Snyder A Z, et al. Spurious but systematic correlations in functional connectivity MRI networks arise from subject motion. NeuroImage, 2012, 59: 2142-2154

11 Zeng L L, Wang D, Fox M D, et al. Neurobiological basis of head motion in brain imaging. Proc Natl Acad Sci USA, 2014, 111: 6058-6062

12 Zuo N, Cheng J, Jiang T. Diffusion magnetic resonance imaging for Brainnetome: A critical review. Neurosci Bull, 2012, 28: 375-388

13 Xie S, Chen L, Zuo N, et al. DiffusionKit: A light one-stop solution for diffusion MRI data analysis. J Neurosci Methods, 2016, 273: 107-119

14 Timmers I, Roebroeck A, Bastiani M, et al. Assessing microstructural substrates of white matter abnormalities: A comparative study using DTI and NODDI. PLoS ONE, 2016, 11: e0167884

15 Barth M, Breuer F, Koopmans P J, et al. Simultaneous multislice (SMS) imaging techniques. Magn Reson Med, 2016, 75: 63-81

16 Huber L, Handwerker D A, Jangraw D C, et al. High-resolution CBV-fMRI allows mapping of laminar activity and connectivity of cortical input and output in human M1. Neuron, 2017, 96: 1253-1263.e7

17 Poplawsky A J, Fukuda M, Murphy M, et al. Layer-specific fMRI responses to excitatory and inhibitory neuronal activities in the olfactory bulb. J Neurosci, 2015, 35: 15263-15275

18 Vandenberghe S, Marsden P K. PET-MRI: A review of challenges and solutions in the development of integrated multimodality imaging. Phys Med Biol, 2015, 60: R115-R154

19 Huster R J, Debener S, Eichele T, et al. Methods for simultaneous EEG-fMRI: An introductory review. J Neurosci, 2012, 32: 6053-6060

20 Lei X, Qiu C, Xu P, et al. A parallel framework for simultaneous EEG/fMRI analysis: Methodology and simulation. NeuroImage, 2010, 52: 1123-1134

21 Gloor P. Hans Berger and the discovery of the electroencephalogram. Electroencephalogr Clin Neurophysiol, 1969, 28:1-36

22 Cohen D. Magnetoencephalography: Evidence of magnetic fields produced by alpha-rhythm currents. Science, 1968, 161: 784-786

23 da Silva F L. EEG and MEG: Relevance to neuroscience. Neuron, 2013, 80: 1112-1128

24 Sejnowski T J, Paulsen O. Network oscillations: Emerging computational principles. J Neurosci, 2006, 26: 1673-1676

25 Fries P. Neuronal gamma-band synchronization as a fundamental process in cortical computation. Annu Rev Neurosci, 2009, 32: 209-224

26 Fries P, Nikolić D, Singer W. The gamma cycle. Trends Neurosci, 2007, 30: 309-316

27 Uhlhaas P J, Singer W. Neuronal dynamics and neuropsychiatric disorders: Toward a translational paradigm for dysfunctional large-scale networks. Neuron, 2012, 75: 963-980

28 Yizhar O, Fenno L E, Prigge M, et al. Neocortical excitation/inhibition balance in information processing and social dysfunction. Nature, 2011, 477: $171-178$

29 Jiang T. Brainnetome: A new -ome to understand the brain and its disorders. NeuroImage, 2013, 80: 263-272

30 Sporns O, Tononi G, Kötter R. The human connectome: A structural description of the human brain. PLoS Comp Biol, 2005, 1: e42

31 Kelly C, Biswal B B, Craddock R C, et al. Characterizing variation in the functional connectome: Promise and pitfalls. Trends Cogn Sci, 2012, 16: $181-188$

32 Van Essen D C. Cartography and connectomes. Neuron, 2013, 80: 775-790

33 Evans A C, Janke A L, Collins D L, et al. Brain templates and atlases. NeuroImage, 2012, 62: 911-922

34 Toga A W, Thompson P M, Mori S, et al. Towards multimodal atlases of the human brain. Nat Rev Neurosci, 2006, 7: 952-966

35 Brodmann K. Vergleichende Lokalisationslehre der Großhirnrinde in ihren Prinzipien dargestellt auf Grund des Zellenbaues. Leipzig (Germany): Verlag von Johann Ambrosius Barth, 1909

36 Von Economo C F and Koskinas G N. Die cytoarchitektonik der hirnrinde des erwachsenen menschen. Berlin: Springer, 1925

37 Talairach J and Tournoux P. Co-planar stereotaxic atlas of the human brain. New York: Thieme, 1988

38 Eickhoff S B, Stephan K E, Mohlberg H, et al. A new SPM toolbox for combining probabilistic cytoarchitectonic maps and functional imaging data. NeuroImage, 2005, 25: 1325-1335

39 Fan L, Li H, Zhuo J, et al. The human brainnetome atlas: A new brain atlas based on connectional architecture. Cereb Cortex, 2016, 26: 35083526 
40 Jiang T, Zhou Y, Liu B, et al. Brainnetome-wide association studies in schizophrenia: The advances and future. Neurosci Biobehav Rev, 2013, 37: 2818-2835

41 Li H, Fan L, Zhuo J, et al. ATPP: A pipeline for automatic tractography-based brain parcellation. Front Neuroinform, 2017, 11: 35

42 Paxinos G. Human brainnetome atlas: A new chapter of brain cartography. Sci China Life Sci, 2016, 59: 965-967

43 Zhang Y, Fan L, Caspers S, et al. Cross-cultural consistency and diversity in intrinsic functional organization of Broca's Region. NeuroImage, 2017, 150: 177-190

44 Fan L, Jiang T. Mapping underlying maturational changes in human brain. Neurosci Bull, 2017, 33: 478-480

45 Jiang T Z. Brainnetome and related projects. Sci China Life Sci, 2014, 57: 462-466

46 Fan L, Li H, Yu S, et al. Human brainnetome atlas and its potential applications in brain-inspired computing, in brain-inspired computing. In: Amunts K, Grandinetti L, Lippert T, et al., eds. Brain-Inspired Computing. BrainComp 2015. Cetraro, 2015. Cham: Springer, 2016. 1-14

47 Xing X X, Zuo X N. The anatomy of reliability: A must read for future human brain mapping. Sci Bull, 2018, 63: 1606-1607

48 Zuo X N, Xu T, Milham M P. Harnessing reliability for neuroscience research. Nat Hum Behav, 2019, 3: 768-771

49 Zuo X N, He Y, Betzel R F, et al. Human connectomics across the life span. Trends Cogn Sci, 2017, 21: 32-45

50 Yan Z X, Liu X, Tan S P, et al. Developmental cognitive neurosciecne: Functional connectomics agenda for human brain lifespan development. Chin Sci Bull, 2016, 61: 718-727

51 Schaefer A, Kong R, Gordon E M, et al. Local-global parcellation of the human cerebral cortex from intrinsic functional connectivity MRI. Cereb Cortex, 2018, 28: 3095-3114

52 Gordon E M, Laumann T O, Adeyemo B, et al. Generation and evaluation of a cortical area parcellation from resting-state correlations. Cereb Cortex, 2016, 26: 288-303

53 Glasser M F, Coalson T S, Robinson E C, et al. A multi-modal parcellation of human cerebral cortex. Nature, 2016, 536: 171-178

54 Salehi M, Greene A S, Karbasi A, et al. There is no single functional atlas even for a single individual: Functional parcel definitions change with task. NeuroImage, 2020, 208: 116366

55 Tzourio-Mazoyer N, Landeau B, Papathanassiou D, et al. Automated anatomical labeling of activations in SPM using a macroscopic anatomical parcellation of the MNI MRI single-subject brain. NeuroImage, 2002, 15: 273-289

56 Destrieux C, Fischl B, Dale A, et al. Automatic parcellation of human cortical gyri and sulci using standard anatomical nomenclature. NeuroImage, 2010, 53: 1-15

57 Xiao X, Yu X, Zhang Z, et al. Transcranial brain atlas. Sci Adv, 2018, 4: eaar6904

58 Jiang T, He Y, Zang Y, et al. Modulation of functional connectivity during the resting state and the motor task. Hum Brain Mapp, 2004, 22: 6371

59 Salvador R, Suckling J, Coleman M R, et al. Neurophysiological architecture of functional magnetic resonance images of human brain. Cereb Cortex, 2005, 15: 1332-1342

$60 \mathrm{Lu} \mathrm{C} \mathrm{M,} \mathrm{Zhang} \mathrm{Y} \mathrm{J,} \mathrm{Biswal} \mathrm{B} \mathrm{B,} \mathrm{et} \mathrm{al.} \mathrm{Use} \mathrm{of} \mathrm{fNIRS} \mathrm{to} \mathrm{assess} \mathrm{resting} \mathrm{state} \mathrm{functional} \mathrm{connectivity.} \mathrm{J} \mathrm{Neurosci} \mathrm{Methods,} \mathrm{2010,} \mathrm{186:} \mathrm{242-249}$

61 Zhang X, Lei X, Wu T, et al. A review of EEG and MEG for brainnetome research. Cogn Neurodyn, 2014, 8: 87-98

62 He Y, Chen Z J, Evans A C. Small-world anatomical networks in the human brain revealed by cortical thickness from MRI. Cereb Cortex, 2007, 17: 2407-2419

63 Hagmann P, Kurant M, Gigandet X, et al. Mapping human whole-brain structural networks with diffusion MRI. PLoS ONE, 2007, 2: e597

64 Rubinov M, Sporns O. Complex network measures of brain connectivity: Uses and interpretations. NeuroImage, 2010, 52: 1059-1069

65 Fair D A, Dosenbach N U F, Church J A, et al. Development of distinct control networks through segregation and integration. Proc Natl Acad Sci USA, 2007, 104: 13507-13512

66 Supekar K, Musen M, Menon V. Development of large-scale functional brain networks in children. PLoS Biol, 2009, 7: e1000157

67 Gao W, Gilmore J H, Giovanello K S, et al. Temporal and spatial evolution of brain network topology during the first two years of life. PLoS ONE, 2011, 6: e25278

68 Gong G, Rosa-Neto P, Carbonell F, et al. Age- and gender-related differences in the cortical anatomical network. J Neurosci, 2009, 29: 1568415693

69 Tomasi D, Volkow N D. Aging and functional brain networks. Mol Psychiatry, 2012, 17: 549-558

70 Wang L, Li Y, Metzak P, et al. Age-related changes in topological patterns of large-scale brain functional networks during memory encoding 
and recognition. NeuroImage, 2010, 50: 862-872

71 Li Y, Liu Y, Li J, et al. Brain anatomical network and intelligence. PLoS Comput Biol, 2009, 5: e1000395

72 van den Heuvel M P, Stam C J, Kahn R S, et al. Efficiency of functional brain networks and intellectual performance. J Neurosci, 2009, 29: $7619-7624$

73 Zuo N, Salami A, Yang Y, et al. Activation-based association profiles differentiate network roles across cognitive loads. Hum Brain Mapp, 2019, 40: 2800-2812

74 Zuo N, Yang Z, Liu Y, et al. Core networks and their reconfiguration patterns across cognitive loads. Hum Brain Mapp, 2018, 39: 3546-3557

75 Yan C, Gong G, Wang J, et al. Sex- and brain size-related small-world structural cortical networks in young adults: A DTI tractography study. Cereb Cortex, 2011, 21: 449-458

76 Tian L, Wang J, Yan C, et al. Hemisphere- and gender-related differences in small-world brain networks: A resting-state functional MRI study. NeuroImage, 2011, 54: 191-202

77 Schmitt J E, Lenroot R K, Wallace G L, et al. Identification of genetically mediated cortical networks: A multivariate study of pediatric twins and siblings. Cereb Cortex, 2008, 18: 1737-1747

78 Fornito A, Zalesky A, Bassett D S, et al. Genetic influences on cost-efficient organization of human cortical functional networks. J Neurosci, 2011, 31: 3261-3270

79 Brown J A, Terashima K H, Burggren A C, et al. Brain network local interconnectivity loss in aging APOE-4 allele carriers. Proc Natl Acad Sci USA, 2011, 108: 20760-20765

80 Xu K, Liu Y, Zhan Y, et al. BRANT: A versatile and extendable resting-state fMRI toolkit. Front Neuroinform, 2018, 12: 52

81 Xia M, Wang J, He Y. BrainNet Viewer: A network visualization tool for human brain connectomics. PLoS ONE, 2013, 8: e68910

82 Song X W, Dong Z Y, Long X Y, et al. REST: A toolkit for resting-state functional magnetic resonance imaging data processing. PLoS ONE, 2011, 6: e25031

83 Yan C, Zang Y. DPARSF: A MATLAB toolbox for "pipeline" data analysis of resting-state fMRI. Front Syst Neurosci, 2010, 4: 13

$84 \mathrm{Xu} \mathrm{T}$, Yang Z, Jiang L, et al. A connectome computation system for discovery science of brain. Sci Bull, 2015, 60: 86-95

85 Yan C G, Wang X D, Zuo X N, et al. DPABI: Data processing \& analysis for (resting-state) brain imaging. Neuroinformatics, 2016, 14: 339-351

86 Dong L, Li F, Liu Q, et al. MATLAB toolboxes for reference electrode standardization technique (REST) of scalp EEG. Front Neurosci, 2017, 11: 601

87 Delorme A, Makeig S. EEGLAB: An open source toolbox for analysis of single-trial EEG dynamics including independent component analysis. J Neurosci Methods, 2004, 134: 9-21

88 Dong L, Luo C, Liu X, et al. Neuroscience information toolbox: An open source toolbox for EEG-fMRI multimodal fusion analysis. Front Neuroinform, 2018, 12: 56

89 Sporns O. Networks of the Brain. Cambridge: MIT Press, 2011

90 Bullmore E, Sporns O. The economy of brain network organization. Nat Rev Neurosci, 2012, 13: 336-349

91 van den Heuvel M P, Mandl R C W, Stam C J, et al. Aberrant frontal and temporal complex network structure in schizophrenia: A graph theoretical analysis. J Neurosci, 2010, 30: 15915-15926

92 Grady C. The cognitive neuroscience of ageing. Nat Rev Neurosci, 2012, 13: 491-505

93 Zuo N, Salami A, Liu H, et al. Functional maintenance in the multiple demand network characterizes superior fluid intelligence in aging. Neurobiol Aging, 2020, 85: 145-153

94 Nyberg L, Lövdén M, Riklund K, et al. Memory aging and brain maintenance. Trends Cogn Sci, 2012, 16: 292-305

95 Sala-Llonch R, Bartrés-Faz D, Junqué C. Reorganization of brain networks in aging: A review of functional connectivity studies. Front Psychol, 2015, 6: 663

96 Cole M W, Reynolds J R, Power J D, et al. Multi-task connectivity reveals flexible hubs for adaptive task control. Nat Neurosci, 2013, 16: 13481355

97 Michalareas G, Vezoli J, van Pelt S, et al. Alpha-beta and gamma rhythms subserve feedback and feedforward influences among human visual cortical areas. Neuron, 2016, 89: 384-397

98 Zhou Y, Fan L, Qiu C, et al. Prefrontal cortex and the dysconnectivity hypothesis of schizophrenia. Neurosci Bull, 2015, 31: 207-219

99 Liu Y, Yu C, Zhang X, et al. Impaired long distance functional connectivity and weighted network architecture in Alzheimer's disease. Cereb 
Cortex, 2014, 24: 1422-1435

100 Zhan Y, Yao H, Wang P, et al. Network-based statistic show aberrant functional connectivity in Alzheimer's disease. IEEE J Sel Top Signal Process, 2016, 10: 1182-1188

101 Zuo N, Fang J, Lv X, et al. White matter abnormalities in major depression: A tract-based spatial statistics and rumination study. PLoS ONE, 2012, 7: e37561

102 Jiang J, Zhu W, Shi F, et al. Thick visual cortex in the early blind. J Neurosci, 2009, 29: 2205-2211

103 Liu Y, Yu C, Liang M, et al. Whole brain functional connectivity in the early blind. Brain, 2007, 130: 2085-2096

104 Feng F, Wang P, Zhao K, et al. Radiomic features of hippocampal subregions in Alzheimer's disease and amnestic mild cognitive impairment. Front Aging Neurosci, 2018, 10: 290

105 Sui J, Qi S, van Erp T G M, et al. Multimodal neuromarkers in schizophrenia via cognition-guided MRI fusion. Nat Commun, 2018, 9: 3028

106 Nielsen J A, Zielinski B A, Fletcher P T, et al. Multisite functional connectivity MRI classification of autism: ABIDE results. Front Hum Neurosci, 2013, 7: 599

107 Peng X, Lin P, Zhang T, et al. Extreme learning machine-based classification of ADHD using brain structural MRI data. PLoS ONE, 2013, 8: e79476

108 Song M, Yang Y, He J, et al. Prognostication of chronic disorders of consciousness using brain functional networks and clinical characteristics. Elife, 2018, 7

109 Wang L, Su L, Shen H, et al. Decoding lifespan changes of the human brain using resting-state functional connectivity MRI. PLoS ONE, 2012, 7: e44530

110 Luo Z, Hou C, Wang L, et al. Gender identification of human cortical 3-D morphology using hierarchical sparsity. Front Hum Neurosci, 2019, 13: 29

111 Jacobs J, Staba R, Asano E, et al. High-frequency oscillations (HFOs) in clinical epilepsy. Prog Neurobiol, 2012, 98: 302-315

112 Bai Y, Xia X, Li X, et al. Spinal cord stimulation modulates frontal delta and gamma in patients of minimally consciousness state. Neuroscience, 2017, 346: 247-254

113 Iadecola C. The neurovascular unit coming of age: A journey through neurovascular coupling in health and disease. Neuron, 2017, 96: 17-42

114 Jiang T, Zuo N, Zhang X, et al. Method for storing data of photoelectrically synchronous brain activity recording. US Patent 10,460,833. 2019 Oct 29

115 Si J, Zhang X, Zhang Y, et al. Hemispheric differences in electrical and hemodynamic responses during hemifield visual stimulation with graded contrasts. Biomed Opt Express, 2017, 8: 2018-2035

116 Akçakaya M, Moeller S, Weingärtner S, et al. Scan-specific robust artificial-neural-networks for k-space interpolation (RAKI) reconstruction: Database-free deep learning for fast imaging. Magn Reson Med, 2019, 81: 439-453

117 Liu Q, Yang Q, Cheng H, et al. Highly undersampled magnetic resonance imaging reconstruction using autoencoding priors. Magn Reson Med, 2020, 83: 322-336

118 Cooley C Z, Stockmann J P, Armstrong B D, et al. Two-dimensional imaging in a lightweight portable MRI scanner without gradient coils. Magn Reson Med, 2015, 73: 872-883

119 Ren Z H, Mu W C, Huang S Y. Design and optimization of a ring-pair permanent magnet array for head imaging in a low-field portable MRI system. IEEE Trans Magn, 2019, 55: 1-8

120 Si J, Zhao R, Zhang Y, et al. A portable fNIRS system with eight channels. In: SPIE BiOS. San Francisco, 2015

121 Yang G R, Joglekar M R, Song H F, et al. Task representations in neural networks trained to perform many cognitive tasks. Nat Neurosci, 2019, 22: 297-306 


\title{
Multi-modal human brain mapping and Brainnetome study
}

\author{
ZUO NianMing ${ }^{1,2} \&$ JIANG TianZi $i^{1,2}$ \\ 1 Brainnetome Center, Institute of Automation, Chinese Academy of Sciences, Beijing 100190, China; \\ 2 National Laboratory of Pattern Recognition, Institute of Automation, Chinese Academy of Sciences, Beijing 100190, China
}

With the development of neuroimaging and computing technologies, brain research based on in vivo imaging attracts mounting interests from multidisciplinary scientists with a variety of backgrounds, including neuroscience, clinical medicine, psychology, and engineering. Recently, cross-discipline collaborative research has formed new research directions and fields, and by contrasting to other research fields, this is a brilliant and unique feature in the development of brain science. Many imaging techniques for characterizing anatomical structures and detecting functional information in the brain have been populated in both basic neuroscience and clinical applications, including magnetic resonance imaging (MRI), electroencephalograph (EEG), magnetoencephalography (MEG). Meanwhile, brain science research puts higher requirements on advanced imaging techniques with higher temporal-spatial resolutions. Consequently, the data produced poses more challenges in data mining and modeling in deciphering the brain. To better describe the strategy of brain research from multiple spatiotemporal scales, the concept of Brainnetome was proposed. This paper systematically reviewed the current representative techniques of imaging-based brain research and emphasized the applications of multi-modal technologies in translational medicine, which is the research background and connotation of Brainnetome. Finally, we made a prospect for the future development of Brainnetome research.

\section{Brainnetome, Brainnetome atlas, brain network, neuroimaging, translational medicine}

doi: $10.1360 / \mathrm{SSV}-2019-0228$ 